\title{
Smart Glasses: A semantic fisheye view on tiled user interfaces
}

\author{
Ilyasse Belkacem, Isabelle Pecci, Benoît Martin \\ LCOMS, Université de Lorraine \\ Ile du Saulcy, CS 50128 \\ 57045 Metz Cedex 01, France \\ Email: \{ilyasse.belkacem, isabelle.pecci, benoit.martin\} \\ @univ-lorraine..fr\}
}

\begin{abstract}
With the evolution of mobile technology, many devices are introduced with very limited screen sizes like smart glasses. This technology must be accompanied with new visualization techniques. A classic interface can't meet the expectations of the user who becomes increasingly hard to please. The challenge is to display information and allow the user a better navigation with less effort especially in situation of mobility. This paper explores a fisheye view on tiled user interfaces for smart glasses that uses the semantic relationships of items of information contained in the tiles. We propose a reformulation of the degree of interest function and a semantic model for tiled interfaces that supports this reformulation. We developed a prototype to demonstrate the feasibility of our approach and to improve our design approach in our future work.

keywords - smartglasses, information visualization, semantic fisheye view, degree of interest
\end{abstract}

\section{INTRODUCTION}

$\mathrm{S}_{\mathrm{i}}$ MART glasses are glasses with a wearable technology, including advanced electronic and IT components (embedded processor, display screen, sensors, camera ..). They allow the visualization and interaction with a large information space. Ergonomics, user-friendly interface and comfortable viewing should be made on these glasses to minimize the time and effort provided by the user during navigation. As smart glasses offer many services, it is necessary to find a display mode that convinces the user by giving him easy access to any service with more information.

The concept of tiles [1] allows the creation of a customized user interface with high flexibility [2]. In use, if we need to make changes to configure the tiles for the user, adjusting the position and size will be easily implemented according to our need. The tiles can contain the services provided by smart glasses, a service can be graphics, text or other visual data. Also, the user can point to a tile and make it active. In addition, the tiles can provide users with fast and direct access to launch the services available through the mobile device [1][2].

We consider that the concept of tiles is suitable for smart glasses. However, a large number of tiles (i.e. services) cause some problems. First of all, it is very difficult to represent all that information on very limited space like the screen of smart glasses and navigate easily. In addition, we cannot link the services together easily if we cannot see all the tiles in the same view. It is also very important to have the global view of services and bind them.

We can overcome these problems with the different techniques that address the access to large data spaces on a limited display area [3] but efficient solution is needed.

In this paper, we present a new concept for a user interface on smart glasses based on the concept of tiles and visualization techniques from a wide data space on a small screen. The rest of the paper is organized as follows. We present in the next section (section 2) the fisheye technique and a related works to our work.

In Section 3 we present our approach and applying it after to a prototype in section 4 . We expose after perspectives and our future work that we consider interesting in section 5 and we will finish with section 6 that will conclude our work.

\section{STATE OF THE ART}

\section{A. Presentation techniques for large data spaces}

There are different techniques of presentation and visualization for large data spaces. They can be categorized into two main techniques: distortion-oriented and nondistortion-oriented [3].

The technique of non-distortion can display some information with scrolling or paging to access to the rest of the information. This technique is less effective if the data space is very large, because the user can be lost during navigation.

On the other hand, distortion technique allows the user to simultaneously visualize a local part with a high level of detail, and to have a vision of the global context with less detail on the same screen. In this technique, there are different types of deformations available that differ in their transformation function [3]: polyfocal display, bifocal display, fisheye view and perspective wall.

Fisheye view technique is the technique adopted by our approach. We will focus on this technique in the following section. The strength of this technique is based on the degree of interest (DOI) that measures the interest of each element of information to present to the user. It enables to present the relevant information in detail and irrelevant information with less detail. 


\section{B. Fisheye view}

The fisheye view is initially proposed by Furnas 1986 [4]. Like other distortions techniques mentioned in the previous section this technique can give a detailed view while keeping the global context. This technique is naturally useful because it is based on the importance and relevance of the information presented to the user.

This technique is inspired by the fisheye lens camera that magnifies near objects and reduces distant objects for a local view in detail and a view of the global context.

\section{DOI function:}

The fisheye technique uses the degree of interest (DOI) function given by:

$$
D O I_{\text {fisheye }}\left(i \mid .=f_{u}\right)=A P I(i)-D\left(i, f_{u}\right)
$$

where $D O I_{\text {fisheye }}$ is the degree of interest for a user to an element $i$ given that the current focus element is $f_{u}$.

The degree of interest function assigns to each item $i$ of the information a value composed of A Priori Importance $A P I(i)$ of the item $i$ and the distance $D\left(i, f_{u}\right)$ between the item $i$ and the current focus item $f_{u}$. The importance API(i) is static and doesn't depend on the current focus item.

The value of the degree of interest increases with the importance and decreases with distance. We can set a threshold $k$ and display only the information items that have a higher degree of interest than the threshold $D_{\text {OI }} I_{\text {fisheye }}\left(i \mid .=f_{u}\right) \geq k$.

This technique was extended by Sarkar and Brown 1992 [5] defining the mathematical formulas for graphics applications. They proposed four functions: the position, size, the amount of detail to display and visual worth of each vertex of a graph.

\section{Emphases algorithm:}

The degree of interest function of Furnas quantifies just the importance of each item of information but without detailing how to present it. It is important for the user to distinguish between the different degrees of interest visually. Emphases algorithm makes the mapping between the degree of interest attributed to an item of information and its encoding in graphics visualization variables such as size, color..

Many techniques exist in the literature. Noik [6] has distinguished 4 types:

- Implicit: an order in the placement of the items, for example. It is generally static.

- Filtered: the items that have a lower degree of interest than the threshold will be filtered.

- Distorted: this technique deforms the size, shape, and position.

- $\quad$ Adorned: emphasizes an item of information using other graphic variables such as color, thickness ...

Many applications used the fisheye view was developed but few of these that integrates semantics. Research work related to our problem is the semantic zoom introduced in several applications such as Pad ++ [11]. It displays more details on an item of information according to the meaning.
The combination of semantic zoom and fisheye technique was introduced in some research work. Zizi and BeaudouinLafon (1995) [12] used the web of documents and information retrieval techniques to provide an interactive map. Van Ham and Van Wijk (2004) [13] used a clustering algorithm to represent semantical distortions for interactive visualization of small world graphs. Janecek \& Pu (2002, 2005) [14] [15] developed a framework for a flight itinerary using relationships between itineraries. Our research has a similar goal of using semantic in a fisheye view with a different approach that we present in the next section for tiled user interface on a promising area application: smart glasses.

\section{PROPOSED APPROACH}

We propose in this section a reformulation of the degree of interest function of the fisheye proposed by Furnas[4] and transform the a priori importance $A P I(i)$ to that takes into account the current point of focus. We also propose an approach that uses this function to compute the degree of interest based on a rich semantic model that considers several concepts.

\section{A. DOI function}

The new proposed formula of the degree of interest is given by:

$$
D O I_{\text {fisheye }}\left(i \mid .=f_{u}\right)=A P I\left(i, f_{u}\right)-D\left(i, f_{u}\right)
$$

where $D O I_{\text {fisheye }}$ is the degree of interest for a user to an item $i$ given that the current focus item is $f_{u}$.

Unlike the Furnas formula cited above, the API of an information item is not static. It depends of the current user item focus. In other words, the importance of an item of information $i$ will be dynamic over the item of current focus $f_{u}$. It does not have the same importance if the user point out two different items.

The main purpose of this reformulation is to provide useful additional information under certain user focus conditions. Thus, the user could discover new items which are strongly linked to the current focus item. This formula can also increase the usability; the user will discover surprising and interesting information which will be useful for him.

We expect that users using a fisheye interface based on this formula can achieve their tasks faster than a using typical fisheye based on previous formula of Furnas.

The DOI function is typically used for hierarchical data structures, structured text, calendars. We want to apply this function to a tiled interface for our smart glasses. Through our approach, we want to access to tiles with a semantic view and meaning.

\section{B. Semantic model}

Each tile provides a service to the user; our goal is to magnify the tiles services that are semantically related when the user hovers on a given tile.

So we need to make comparisons between different services. For this we need a semantic description based on 
the analysis of each service to detect what they have in common in terms of content. We can make this description based on metadata and attributes of these services, but the main challenge is to select attributes that are meaningful to the user.

In figure 1 , we propose a semantic model focused on the service that defines the main concepts that allow computing our measure of semantic similarity between the different services.

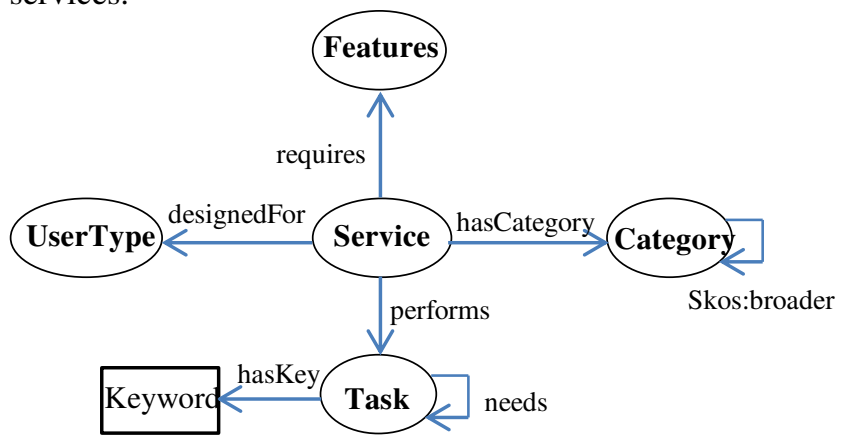

Fig. 1 Semantic service model

Our semantic model uses 4 concepts:

- Category: modeled by a tree structure, each category can have several sub categories (e.g. multimedia category can have two sub categories: audio and video).

- Task: performed by some services. A service can perform one or more tasks, so two services that have several common tasks to execute are probably similar. The goal is not just to identify similar services, but also services that are connected in some way to a service.

- User type: Two services can have the same category and perform the same tasks but are not designed for the same type of users.

- Features: A service may require authentication, internet connection and a camera to operate. Such requirement can give meaning to the proximity between the two services.

After building the model, we must define a similarity measure that quantifies a semantic relatedness with a numeric value between two services from our model. The similarity between two services can be defined by common properties. The services that have more common properties are more similar [7].

Each property has a type; it can be organized hierarchically (e.g. Category) or not organized hierarchically (e.g. Tasks) or a literal (we don't have this type in our model). Different measures of semantic proximities according to property nature are present in the literature [8].

Our calculation has to integrate different types of properties to have a single numeric value that represents the similarity between two services. We describe here the similarity measures that we have chosen for each type:

\section{Properties hierarchically organized:}

This type is based on hierarchies of properties. $\mathrm{Wu}$ and Palmer measure [9] uses the depth of the two properties and the depth of the least common subsummer $(l c l)$. It's defined as:

$$
\operatorname{sim}_{W u, \text { Palmer }}\left(p_{1}, p_{2}\right)=\frac{2 \times \operatorname{depth}\left(\operatorname{lcl}\left(p_{1}, p_{2}\right)\right)}{\operatorname{depth}\left(p_{1}\right)+\operatorname{depth}\left(p_{2}\right)}
$$

Properties not hierarchically organized:

The similarity is computed with Jaccard measure [10] which takes into account the number of common properties compared to the total number of properties. It's defined as:

$$
\operatorname{sim}_{\text {Jaccard }}\left(p_{1}, p_{2}\right)=\frac{\left\{p_{11} \ldots p_{12}\right\} \cap\left\{p_{21} \ldots p_{22}\right\}}{\left\{p_{11 \ldots} \ldots p_{12}\right\} \cup\left\{p_{21} \ldots p_{22}\right\}}
$$

where $\left\{p_{11} \ldots p_{12}\right\}$ and $\left\{p_{21} \ldots p_{22}\right\}$ are respectively the values of the proporities $p_{1}$ and $p_{2}$.

After computing a similarity value for each property (hasCategoy, needs, performs, requires). We can make a similarity vector based on these similarities. Each component of the vector contains a similarity value of a property. We need to aggregate this vector into a single value using an aggregate function. We choose the weighted average as function on the hypothesis that the semantic concepts don't have necessarily the same importance.

The similarity between two services $S_{1}$ and $S_{2}$ is given by:

$$
\operatorname{Sim}\left(S_{1}, S_{2}\right)=\frac{\sum_{k} w_{k} \times \operatorname{Sim}_{k}\left(p_{S_{1}}, p_{S_{2}}\right)}{\sum k}
$$

where $w_{k}$ is the weight of the property $p$ for $S_{1}$ and $S_{2}$. And the DOI function becomes:

$$
D O I_{\text {fisheye }}\left(i \mid .=f_{u}\right)=\operatorname{sim}\left(i, f_{u}\right)-D\left(i, f_{u}\right)
$$

\section{A PROTOTYPE: PROOF OF CONCEPT}

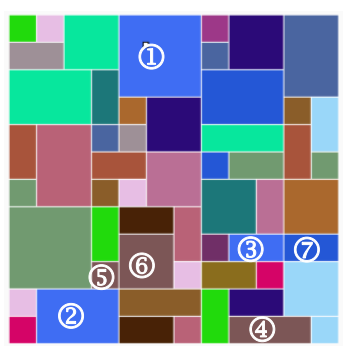

(a) normal view

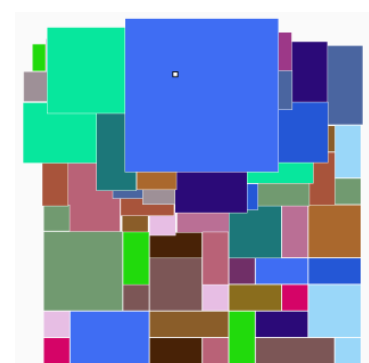

(b) fisheye view

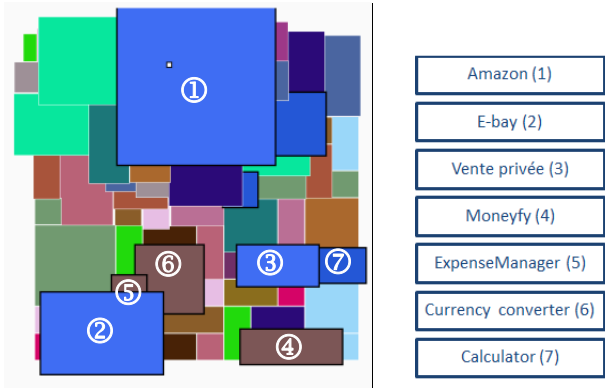

(c) semantic fisheye view

Fig. The proof of concept

Our prototype developed is a tiled interface on android platform for smart glasses. The semantic model is built by Protégé ${ }^{1}$ integrating 56 services, each tile in the interface contain a service. We use Jena ${ }^{2}$ Framework to be able to compute different similarities from the model. We consider

\footnotetext{
${ }^{1}$ https://jena.apache.org/

${ }^{2}$ http://protege.stanford.edu/
} 
in our prototype only the category to compute similarities from the model.

Figure 2.a shows the tiled view without any fisheye view. Figure 2.b takes into account only distance to magnify tiles for a fisheye view.

The semantic model is activated in figure 2.c. The associated service to the tile (1) is Amazon. From the computation in our model, it has a similarity of $\operatorname{sim}=1$ with e-bay (2) and Vente-privee (3) because they belong to the same sub-category Shopping. The services: Monefy (4), ExpenseManager(5), CurrencyConverter (6) and the service: Calculator (7) belong respectively to Banking and Shopping categories whose main category is Business which is also the main category of Shopping. So they have a similarity of $\operatorname{sim}=0.5$ with the Amazon service.

We have taken a similarity threshold $k=0.5$ to emphases a tile. We are aware of the need of building a good emphases algorithm that will be the subject of another work in the future. For this prototype, when the user hovers over a tile, we compute all sizes of tiles to allocate more space for important tiles on relation with the focus item. The size of a tile in the fisheye view depends on the size of the tile in the normal view, the distance from focus tile and the importance of the item that the tile contains. We notice that the tile (3) and (4) had the same size in normal view but was not magnified with the same degree because their services don't have the same similarity with Amazon services.

\section{PERSPECTIVES}

In this paper, we described a reformulation of the degree of interest introduced by Furnas [4] for fisheye view. The importance of an item is dynamic and depends also on the current focus item. Building a semantic model that takes into account the user context (location, time ...) and preferences to determine the degree of interest of an item is our major improvement axis that has a strong impact on the navigation of the user with smart glasses. Also, a more sophisticated aggregate function that replaces the weighted average is necessary for computing the global similarity.

In terms of visualization, fisheye view must support changes in the context and user preferences with flexibility using different emphases techniques.

We are planning an evaluation of our approach through the recruitment of users for an experiment by creating two interfaces: one with a classic fisheye view and the second with our semantic fisheye view to accomplish tasks on smart glasses and make a comparison on different criteria.

\section{CONCLUSION}

Using of wearable devices like smart glasses is continually increasing. Smart glasses have a wide services area on a limited screen size. This involves viewing problems and interaction.

It becomes important to offer carefully designed interfaces that fit with small screens and overcome their limitations in order to improve the user experience. They should help users to easily navigate and understand the information presented to perform their tasks quickly and efficiently.

We aimed to address this problem by proposing a semantic fisheye view on tiled interfaces which allows enriching the user navigation using the relationships between the different services contained in the tiles.

Research on these aspects has a strong impact on the support of the mobile technology and the rise of using smart glasses.

\section{ACKNOWLEDGMENT}

This work was performed within the eGLASSES project, which is partially funded by NCBiR, FWF, SNSF, ANRand FNR under the ERA-NET CHIST-ERAII framework. The authors thank Arthur DOQUET for his help with the development part.

\section{REFERENCES}

[1] Flynt, David Wayne, et al. "Tile space user interface for mobile devices." U.S. Patent No. 7,933,632. 26 Apr. 2011.

[2] Walter, Wolfgang E., and Christoph Persich. "Active Tiled User Interface." U.S. Patent Application No. 11/829,025.

[3] Leung, Y. K., \& Apperley, M. D. (1994). A review and taxonomy of distortion-oriented presentation techniques. ACM Transactions on Computer-Human Interaction (TOCHI), 1(2), 126-160.

[4] Furnas, G. W. (1986). Generalized fisheye views (Vol. 17, No. 4, pp. 16-23). ACM.

[5] Sarkar, M., \& Brown, M. H. (1992, June). Graphical fisheye views of graphs. In Proceedings of the SIGCHI conference on Human factors in computing systems (pp. 83-91). ACM.

[6] Noik, E. G. (1994, May). A space of presentation emphasis techniques for visualizing graphs. In Graphics Interface (pp. 225-225). CANADIAN INFORMATION PROCESSING SOCIETY.

[7] Pirró, G., \& Euzenat, J. (2010). A feature and information theoretic framework for semantic similarity and relatedness. In The Semantic Web-ISWC 2010 (pp. 615-630). Springer Berlin Heidelberg.

[8] Petrakis, E. G., Varelas, G., Hliaoutakis, A., \& Raftopoulou, P. (2006). X-similarity: computing semantic similarity between concepts from different ontologies. JDIM, 4(4), 233-237.

[9] Wu, Z., \& Palmer, M. (1994, June). Verbs semantics and lexical selection. In Proceedings of the 32nd annual meeting on Association for Computational Linguistics (pp. 133-138). Association for Computational Linguistics.

[10] Niwattanakul, S., Singthongchai, J., Naenudorn, E., \& Wanapu, S. (2013, March). Using of Jaccard coefficient for keywords similarity. In Proceedings of the International MultiConference of Engineers and Computer Scientists (Vol. 1, p. 6).

[11] Bederson, B. B., \& Hollan, J. D. (1994, November). Pad++: a zooming graphical interface for exploring alternate interface physics. In Proceedings of the 7th annual ACM symposium on User interface software and technology (pp. 17-26). ACM.

[12] Zizi, M., \& Beaudouin-Lafon, M. (1995). Hypermedia exploration with interactive dynamic maps. International Journal of HumanComputer Studies, 43(3), 441-464.

[13] Van Ham, F., \& Van Wijk, J. J. (2004, October). Interactive visualization of small world graphs. In Information Visualization, 2004. INFOVIS 2004. IEEE Symposium on (pp. 199-206). IEEE.

[14] Janecek, P., \& Pu, P. (2002, May). A framework for designing fisheye views to support multiple semantic contexts. In Proceedings of the Working Conference on Advanced Visual Interfaces (pp. 51-58). ACM.

[15] R Janecek, P., \& Pu, P. (2005). An evaluation of semantic fisheye views for opportunistic search in an annotated image collection. International Journal on Digital Libraries, 5(1), 42-56. 